\title{
Ossification of the Posterior Longitudinal Ligament
}

\author{
Jae-Won Doh, MD, PhD \\ President of Korean Spinal Neurosurgery Society \\ Professor of Neurosurgery, Soonchunhyang University Medical College, Chungnam, Korea
}

The book, Ossification of the Posterior Longitudinal Ligament, has a significant meaning as the first English contribution of comprehensive coverage for OPLL ever published in Korea.

The book covers the whole of epidemiology, natural course and surgical treatment of OPLL; hence, it is indispensable to spine surgeons with particular interest in OPLL. The book was written not only by Korean spine surgeons, but also by Japanese and Taiwanese spine surgeons, who all have acclaimed expertise in OPLL. With this diversity of contributors, the book benefited from the prevalence of OPLL covering the entire East Asian ethnic groups, including Korean, Japanese and Chinese, if we could dare to represent the whole Chinese population in several published studies.

I noticed a very interesting finding in the book, which described that the neurological deterioration rate is similar between the surgical group and the non-surgical group, as shown in the survey of surgical operations (387 cases) performed at Seoul National University Hospital. This result suggests that we, spine surgeons, need to refine the surgical indication and to better hone our surgical technique by taking the progressive nature of OPLL into account.

I wish that this book would encompass more epidemiological studies in China. OPLL is prevalent in East Asia and further, China is the most populated country; thus, it could provide a vast amount of clinical data and experiences. I anticipate that such data will be added to the next edition, which would make the epidemiologic picture of OPLL more complete.

Lastly, along with all the members of the Korean Spinal Neurosurgery Society (KSNS), I would like to congratulate the publication of this book and express my sincere gratitude to Professor Hyun Jib Kim and Professor Chun Kee Chung for their contribution to these pressing health issues which are now placing more burdens to patients as well as to spine sur- geons. 